\title{
Asymmetric distribution of mitochondria and of spindle microtubules in opposite directions in differential mitosis of germ line cells in Acricotopus
}

\author{
Wolfgang Staiber
}

Received: 14 November 2006 / Accepted: 5 February 2007 / Published online: 20 March 2007

(C) Springer-Verlag 2007

\begin{abstract}
Additional chromosomes present only in the germ line are a specific feature of the Orthocladiinae, a subfamily of the Chironomidae. During the complex chromosome cycle in the orthocladiid Acricotopus lucidus, about half of the germ-line-limited chromosomes (Ks) are eliminated in the first division of the primary germ cells. Following normal gonial mitoses, the reduction in the number of Ks is compensated for, in the last mitosis prior to meiosis, by a monopolar movement of the unseparated Ks, while the somatic chromosomes (Ss) segregate equally. This differential mitosis produces daughter cells with different chromosome constitutions and diverse developmental fates. A preferential segregation of mitochondria occurs to one pole associated with an asymmetric formation of the mitotic spindle. This has been detected in living gonial cells in both sexes by using MitoTracker probes and fluorochrome-labelled paclitaxel (taxol). In males, the resulting unequal partitioning of mitochondria to the daughter cells is equalised by the transport of mitochondria through a permanent cytoplasmic bridge from the aberrant spermatocyte to the primary spermatocyte. This asymmetry in the distribution and in the segregation of cytoplasmic components in differential gonial mitosis in Acricotopus may be involved in the process of cell-fate determination.
\end{abstract}

Electronic supplementary material The online version of this article (doi:10.1007/s00441-007-0400-z) contains supplementary material, which is available to authorised users.

W. Staiber $(\bowtie)$

Institute of Genetics, University of Hohenheim,

Garbenstrasse 30,

70599 Stuttgart, Germany

e-mail: wstaiber@uni-hohenheim.de
Keywords Asymmetric cell division · Differential mitosis · Germ-line-limited chromosomes · Mitochondria · Mitotic spindle $\cdot$ Acricotopus lucidus (Diptera, Chironomidae)

\section{Introduction}

In a normal mitosis, both daughter cells usually receive identical sets of chromosomes and genes. One exception to this rule is the monopolar movement of unseparated germ-line-limited chromosomes (Ks; $\mathrm{K}$ being derived from "Keimbahn"; Bauer 1970) during the last gonial mitosis prior to meiosis of young fourth instar larvae in both sexes of the dipteran Acricotopus lucidus (Chironomidae, Orthocladiinae; Bauer and Beermann 1952). This unequal, so-called differential, mitosis doubles the number of Ks in the germ line and so compensates for the elimination of about half of the Ks in the first gonial mitosis of the primary germ cells (for reviews of the chromosome cycle, see White 1973; Redi et al. 2001). In contrast to the Ks, the somatic chromosomes (Ss) separate equally in differential mitosis. This special mitosis produces daughter cells with different chromosome constitutions and diverse developmental fates. The cells receiving all the Ks and two $\mathrm{S}$ sets develop into oocytes and into primary spermatocytes, whereas the cells receiving only two $\mathrm{S}$ sets differentiate into a nurse cell and an aberrant spermatocyte. The single nurse cell undergoes endomitotic cycles and becomes highly polyploid. The aberrant spermatocyte doubles its chromosomes prior to meiosis but does not engage in meiotic divisions in the same way as the primary spermatocyte.

The differential gonial mitosis in Acricotopus is a case of asymmetric cell division generating daughter cells with different developmental fates (Horvitz and Herskowitz 
1992; Kaltschmidt and Brand 2002). An asymmetric distribution of cell-fate determinants along the mitotic spindle axis in the mother cell, followed by an unequal partitioning of the determinants to the daughter cells, can generate cells with different fates, as is known in Drosophila neuroblast differentiation (Bellaiche et al. 2001; Betschinger and Knoblich 2004).

In the present study, the spatial arrangement of cytoplasmic components was analysed in differential gonial mitosis of Acricotopus. A preferential segregation of mitochondria to one cell pole and an asymmetric distribution of spindle microtubules (MTs) were detected, and this may be involved in the process of cell-fate determination in the germ line of Acricotopus.

\section{Materials and methods}

\section{Animals and living cell imaging}

The animals used in this study came from the laboratory stock of Acricotopus lucidus (Diptera, Chironomidae) kept in our Institute. For imaging of living gonial cells, ovaries and testes from 4th instar larvae and from prepupae were dissected on a cover glass in a drop of a 1:1 mixture of Ringer's solution $(100 \mathrm{mM} \mathrm{NaCl}, 3 \mathrm{mM} \mathrm{KCl}, 3 \mathrm{mM}$ $\mathrm{CaCl}_{2}, \mathrm{pH}$ 7.2), and Shields and Sang M3 insect medium (Sigma), supplemented with $10 \%$ fetal calf serum and $50 \mu \mathrm{g} / \mathrm{ml}$ gentamycin (Sigma). The drop was then contacted with a glass slide inducing the attraction of the cover glass and producing slight compression of the gonial cells. Preparations were analysed at room temperature by phase-contrast microscopy with a Planapochromat $63 \times / 1.4$ oil objective (Zeiss).

\section{Fluorescence microscopy}

Fluorescence labelling of live mitochondria was obtained by incubating the gonial cells in the above 1:1 media mixture containing $0.2-0.4 \mu \mathrm{M}$ MitoTracker $\mathrm{R}$ red CMXRos (Molecular Probes) for about 5-10 min. For the simultaneous visualisation of tubulin, mitochondria and DNA, gonial cells were placed for about $10 \mathrm{~min}$ in the above medium mix, without fetal calf serum but containing $1 \mu \mathrm{M}$ Oregon Green 488 paclitaxel (Molecular Probes), 0.2-0.4 $\mu \mathrm{M}$ MitoTracker $\mathrm{R}$ red CMXRos (Molecular Probes), $1 \mu \mathrm{g} / \mathrm{ml}$ 4',6'-diamidino-2-phenylindole (DAPI; Serva) and $0.025 \%$ Triton X-100. Fluorescence images were captured with a Canon A 80 camera attached to an epifluorescence microscope (Zeiss), and a Neofluar $100 \times / 1.30$ oil objective. Digital images were combined and pseudocoloured by using a Corel Draw software package.

\section{Results}

In the first mitosis of the primary germ cells in the gonads of young first instar larvae of Acricotopus, about half of the $\mathrm{Ks}$ are eliminated by remaining in the equatorial plane (Bauer and Beermann 1952). The following gonial mitoses (Fig. 1a) proceed normally and, thus, each of the polewards-moving chromosome complements consists of two sets of Ss and one set of Ks. Subsequently, during the last gonial mitosis prior to meiosis in young fourth instar larvae, all Ks move as unseparated sister chromatids to one cell pole (lower pole in Fig. 1b), while the Ss first remain in the metaphase plane and separate normally just before the Ks arrive at the pole (see time-lapse imaging of normal gonial mitosis and differential gonial mitosis in Supplemental Materials). The result of such a differential mitosis is two daughter cells with different chromosome constitutions and DNA contents: one has received all Ks $(2 n=12-32$; Staiber $1988)$ and two sets of Ss $(2 n=6)$, whereas the other has received only two $\mathrm{S}$ sets. In the male, these cells differentiate into the K-containing primary spermatocyte and to an aberrant spermatocyte (Fig. 1c), whereas in the female, the cells develop as the oocyte and as one nurse cell (Fig. 1d,e). In both sexes, cytokinesis will not be completed and, therefore, both daughter cells remain connected by a cytoplasmic bridge (arrowheads in Fig. 1c, insert in Fig. 1d). The primary spermatocyte and the oocyte receive smaller amounts of cytoplasm than the aberrant spermatocyte and nurse cell. In interphase, the aberrant spermatocytes and nurse cells each produce one nucleolus, which is formed from the paired nucleolus organiser regions located on the third chromosomes (Staiber and Behnke 1985). The morphology and the size of these syncytial complexes resulting from incomplete cytokinesis are similar in both males and females (cf. Fig. 1c, insert in Fig. 1d). During the following development, an enhanced growth of the nurse cell takes place together with polyploidisation and a size increase of the nucleus and nucleolus (cf. Fig. 1d, insert). In the egg chamber (Fig. 1d) from an old fourth instar larva, the nurse cell and the posterior oocyte are surrounded by a layer of somatic follicle cells forming an appendix at the anterior site. The actual growth of the oocytes begins with the deposition of yolk spheres, which appear as brightly lit particles (Fig. 1e). The polyploidisation of the nurse cell proceeds and can generate amounts of nuclear DNA up to 4096 C in old prepupae of Acricotopus (Thudium 1974).

Phase-contrast images of living gonial cells in differential mitosis and time-lapse video analyses indicate an unequal distribution of mitochondria within the cell during monopolar movement of the Ks. In order to verify this observation, specific fluorescence staining of live mitochondria with the MitoTracker Red marker was applied. The phase-contrast and mitochondrial fluorescence images 


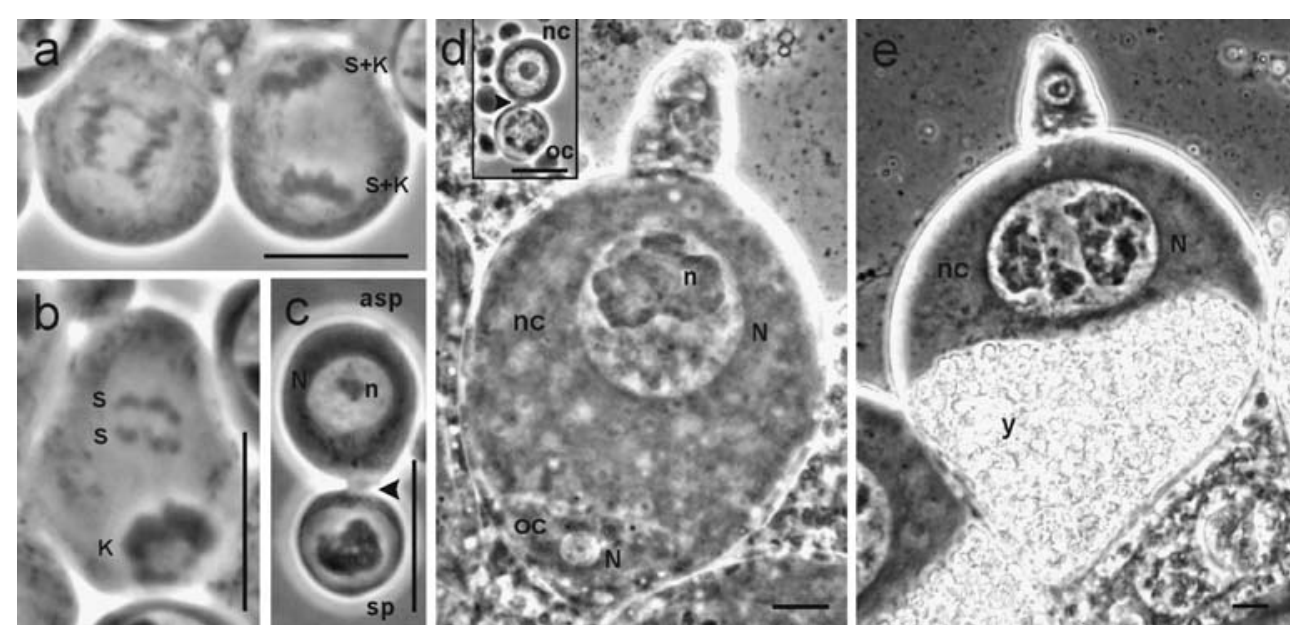

Fig. 1 a, b Regular (a) and differential (b) spermatogonial mitoses in Acricotopus lucidus viewed by phase-contrast microscopy. b In the differential mitosis, all Ks $(K)$ have moved as unseparated sister chromatids to only one pole (lower), while the Ss $(S)$ just segregate equally (see time-lapse videos in Supplemental Materials). c Primary spermatocyte ( $s p ; 2 \mathrm{~K}$ and $2 \mathrm{~S}$ sets) and aberrant spermatocyte (asp; $2 \mathrm{~S}$ sets). The daughter cells ( $N$ nucleus, $n$ nucleolus) of a differential spermatogonial mitosis remain connected through a permanent canal- like cytoplasmic bridge (arrowhead). d Insert: The daughter cells of a differential oogonial mitosis from a young fourth instar larva have the same appearance as those of a differential spermatogonial mitosis (compare with c) but differentiate into the primary oocyte $(o c)$ and to a nurse cell $(n c)$. d, e Egg chambers from a 4th instar larva (d) showing the oocyte $(o c)$ and the polyploid nurse cell $(n c)$ and their nuclei $(N)$ and nucleoli $(n)$, and from a prepupa (e) with a growing mass of yolk particles $(y)$ in the oocyte. Bars $10 \mu \mathrm{m}$ of two spermatogonial differential mitoses and the combined image are presented in Fig. 2a, a',a". During movement of all the Ks to only one cell pole (upper Ks in Fig. 2a), the tubule-like mitochondria accumulate in the opposite half of the cell. The same unequal distribution of mitochondria also occurs during differential mitosis in the female germ line (Fig. 2b, $b^{\prime}$ ). The mitochondria therefore aggregate in the cell half that receives only two $\mathrm{S}$ sets and that then develops into an aberrant spermatocyte or the nurse cell.

Completion of differential mitosis results in an unequal partitioning of mitochondria to the daughter cells. The aberrant spermatocyte receives many more mitochondria than the primary spermatocyte (Fig. 2c). This surplus of mitochondria in the aberrant spermatocyte is then reduced by the transport of mitochondria through the connecting cytoplasmic bridge to the primary spermatocyte (Fig. 2d,e). In normal spermatogonial mitoses, the mitochondria are evenly distributed around the mitotic spindle and in both cell halves (Fig. 2f). They then partition equally to the daughter cells. In interphase spermatogonial cells prior to differential mitosis, the mitochondria often appear to be clustered to one side of the cell, as shown in Fig. $2 \mathrm{~g}$.

The asymmetric arrangement of mitochondria in differential mitosis led to investigations of the arrangement of MTs and of the changes in the organisation of the spindle during this specific mitosis. Visualisation of MTs in living gonial cells was made using tubulin-binding paclitaxel (taxol) labelled with the fluorochrome Oregon Green. Chromosomes were stained with DAPI and pseu- docoloured red (Fig. 3a-n). In testis preparations, clusters of spermatogonial cells, at the same stage of differential mitoses, occurred with the monopolar-moving Ks (Fig. 3a) or with the equally segregating Ss (Fig. 3b). Not only alpha- and beta-tubulin of the MTs, but also gamma-tubulin of the centrioles and, consequently, of the centrosomes could be visualised with paclitaxel, as shown in interphase and metaphase cells (Fig. 3c,d, arrowheads).

In normal spermatogonial mitoses, metaphase cells exhibit equally sized symmetrical half-spindles (Fig. 3d) formed by kinetochore MTs and polar MTs. In anaphase, the $\mathrm{K}$ and $\mathrm{S}$ chromatids segregate equally and the opposite cell poles are pushed apart from each other (anaphase B). In telophase, an increasing fluorescence in the middle of the MT bundles, lying between the daughter nuclei, indicates an accumulation of MTs in the mid-body (Fig. 3e,f). In contrast to normal gonial mitoses, metaphase cells of differential mitoses display only half-spindles with a characteristic unequal distribution and different densities of MTs (Fig. 3g). The position of the metaphase plane within the cell is clearly asymmetrical, with different distances to the spindle poles. During the following monopolar movement of Ks, extensive arrays of shortening MTs extend from the polewards-moving Ks towards the centrosome (Fig. 3h,i). At the same time, bundles of kinetochore MTs of different length extend from the unseparated Ss, which are still arranged in the metaphase plane, towards both poles. Thus, the MTs mainly pass through and are concentrated in the middle of the polewards-moving group of Ks (Fig. 3i). Just before the Ks 

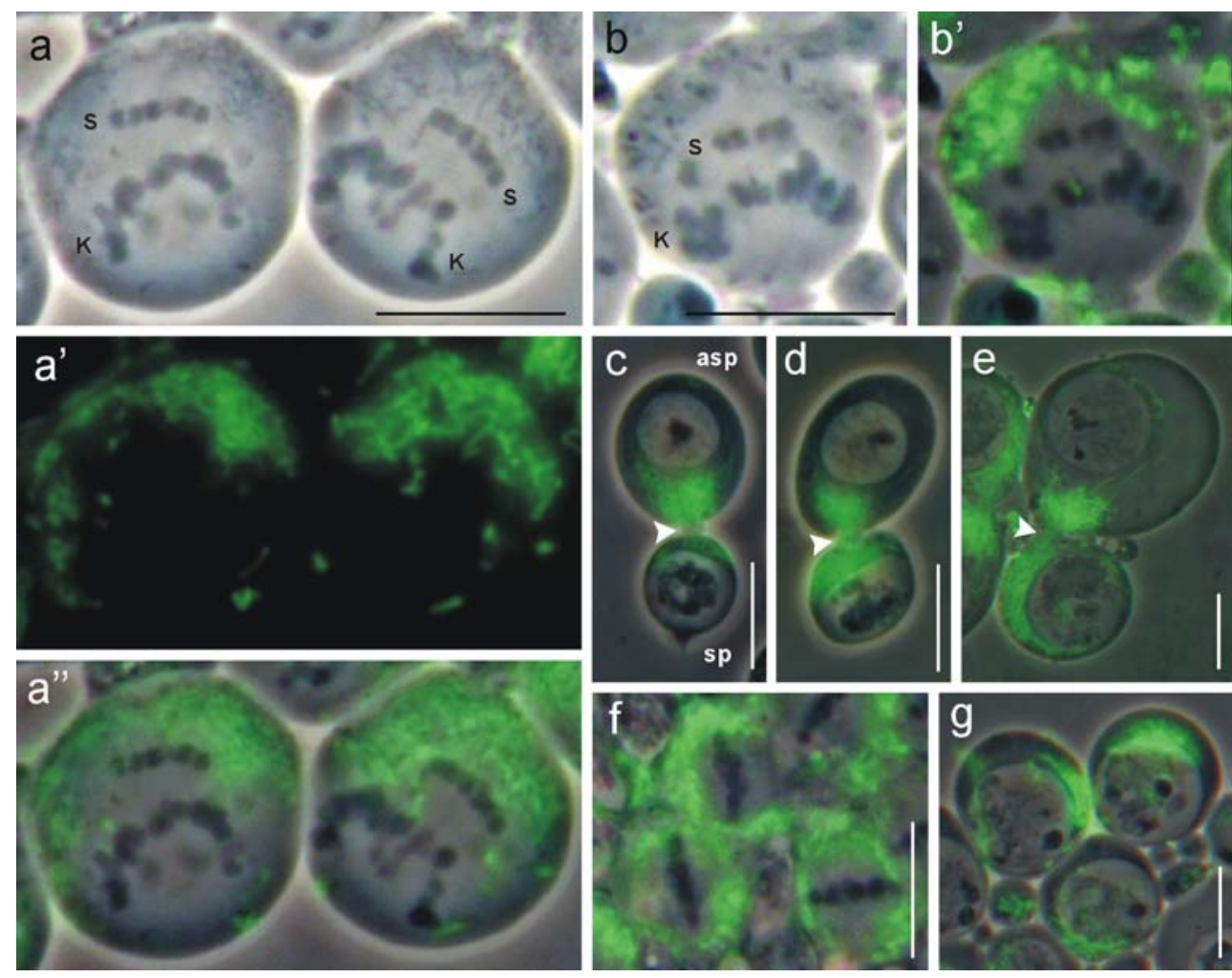

Fig. 2 Analysis of the distribution and arrangement of mitochondria in living gonial cells analysed by MitoTracker selective fluorescence staining. The red fluorescence of mitochondria was pseudocoloured green. $\mathbf{a}, \mathbf{a}^{\prime}, \mathbf{a}^{\prime \prime}$ Asymmetric distribution of mitochondria in differential mitoses in spermatogonial cells of Acricotopus. b, $\mathbf{b}^{\prime}$ Asymmetric distribution of mitochondria in differential mitoses in oogonial cells of Acricotopus. a, b Phase-contrast image. a' Fluorescence image. a", b' Combined

images. c Results of differential mitosis: the aberrant spermatocyte (asp) contains considerably more mitochondria than the primary spermatocyte $(s p)$. d, e Successively more mitochondria from the aberrant spermatocyte are transported through the cytoplasmic bridge (arrowhead) into the spermatocyte. f As a comparison, note the even distribution of mitochondria in normal spermatogonial mitoses. $\mathbf{g}$ Clustered distribution of mitochondria in spermatogonia prior to differential mitosis. Bars $10 \mu \mathrm{m}$

reach the cell pole, the S sister chromatids separate (Fig. 3j; see also time-lapse video of differential mitosis in Supplemental Materials) and start to migrate to the opposite poles (Fig. 3k, anaphase A). At the same time, the polar MTs push the poles apart (Fig. 31, anaphase B). As one S complement arrives at the centrosome (left in Fig. $3 \mathrm{~m}$ ), the other Ss reaches the group of Ks. This latter $\mathrm{S}$ group remains at the periphery of the Ks and does not move to the pole (Fig. 3m). An increasing MT fluorescence in the midzone indicates the developing mid-body. The MTs extend and wind around the nuclei of the primary and the aberrant spermatocyte (Fig. 3n). The asymmetric and oppositely directed arrangement of mitochondria and of MTs during differential mitoses becomes clear when combining the fluorescence images of these cell components together with the DAPI fluorescence image of the chromosomes. The combined images of fluorescence for mitochondria (red), MTs (green), and chromosomes (blue) are shown in Fig. 3o, p. The insert in Fig. 3p represents an interphase cell prior to differential mitosis, with mitochondria (red) concentrated in one cell half.

\section{Discussion}

The polar localisation of cell-fate determinants in the mother cell and their unequal partitioning to the daughter cells are fundamental mechanisms used to establish different cell fates in asymmetric cell division (Betschinger and Knoblich 2004). Polarisation involves the asymmetric distribution of molecules, cytoskeletal structures and organelles within the cell. The process of active segregation and accumulation within the cell is poorly understood but has been shown to depend on the cytoskeleton (Lopez de Heredia and Jansen 2004).

In the germ line of Acricotopus, an asymmetric cell division with determination of diverse cell fates takes place in the last gonial mitosis, accompanied by the differential segregation of Ks, caused by their monopolar movement, to the daughter cells. This differential mitosis is characterised by considerable spindle asymmetry, together with an asymmetric distribution of mitochondria accumulating in that cell half that lies opposite to the polewards-moving Ks. The polar arrangement of mitochondria within a cell can 

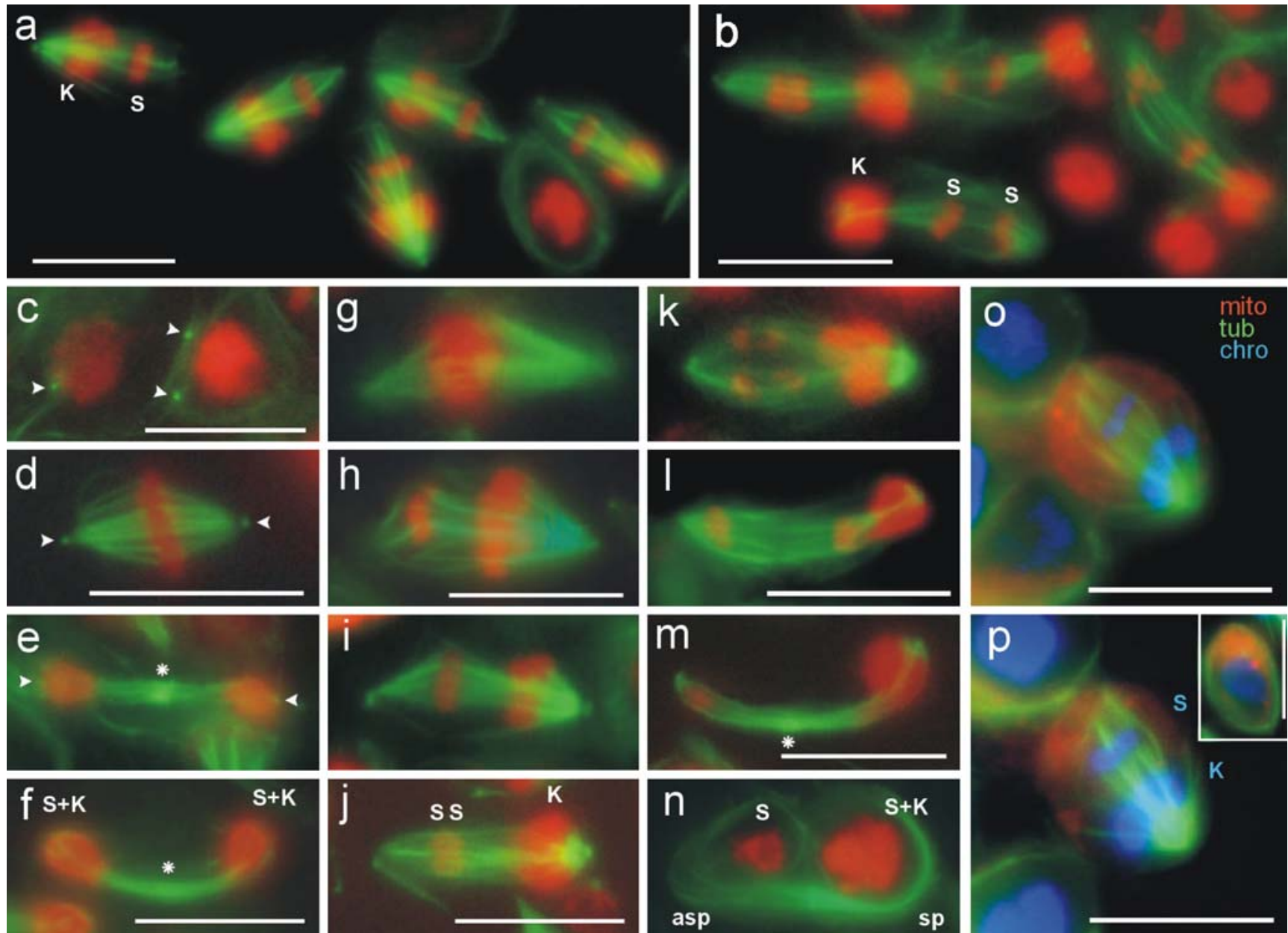

Fig. 3 Visualisation of microtubule (MT) distribution in normal and differential mitoses of living spermatogonial cells of Acricotopus by using fluorescent Oregon-Green-labelled paclitaxel (taxol). DNA was stained with DAPI and pseudocoloured red. a, b Survey of clusters of spermatogonial cells at different stages of differential mitosis. a Monopolar movement of Ks $(K)$. b Equal segregation of Ss $(S)$. c Premitotic cells; the cell right has doubled centrosomes (arrowheads). d-f Normal gonial mitoses. d Metaphase with equally sized half spindles and polar centrosomes. e, f Telophases with arising midbodies (asterisks). $\mathbf{g}-\mathbf{m}$ Successive stages of differential mitoses. Unequal half spindles present in metaphase (g) and during monopolar

result either from an active transport of mitochondria along MTs to one cell half (Hollenbeck and Saxton 2005) or from a passive counter-current displacement of mitochondria out of that cell half, forming a more expanded half-spindle with higher MT density. In addition, the clustering of mitochondria at one side of the cell observed in interphase spermatogonial cells, prior to differential mitoses (Figs. $2 \mathrm{~g}$, 3p, insert), supports the idea of an active polar accumulation of mitochondria. This indicates that the polar and asymmetric arrangement of mitochondria may be involved in the determination of the spindle axis and of the fates of the daughter cells (Kaltschmidt and Brand 2002). Another case of the asymmetric segregation of mitochondria has been reported
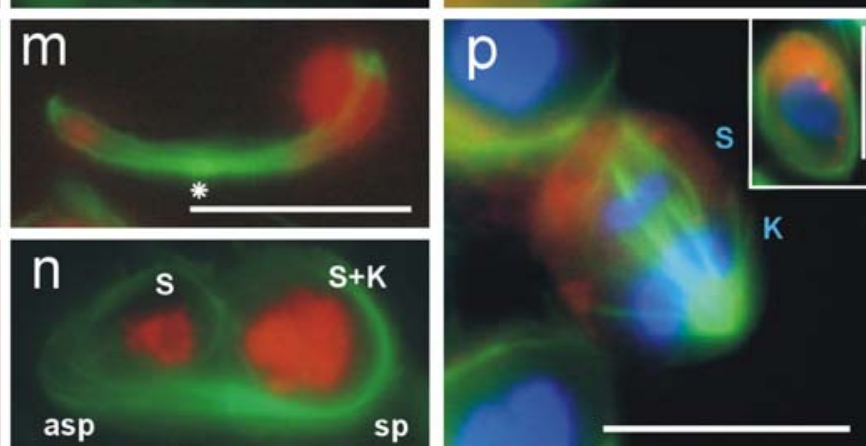

asp sp

movement $(\mathbf{h}, \mathbf{i})$ of Ks. $\mathbf{j}-\mathbf{l}$ Equal segregation and oppositely directed anaphasic movement of $\mathrm{S}$ sister chromatids $(S)$. m Telophase with increasing MT fluorescence in region of the mid-body (asterisk). n Dense bundles of MTs pass through the cytoplasmic bridge connecting primary spermatocyte ( $s p, r i g h t)$ and aberrant spermatocyte (asp, left). $\mathbf{o}, \mathbf{p}$ Combined fluorescence images of MTs (green), mitochondria (red) and chromosomes (blue) of differential spermatogonial mitoses at the time of monopolar movement of Ks and, in the insert in p, of a gonial cell prior to differential mitosis ( $K$ germ-line-limited chromosomes, $S$ somatic chromosomes). Bars $10 \mu \mathrm{m}$

for the asymmetric division of sensory cell progenitors, which are common precursors of hair cells and supporting cells in the inner ear (Rivolta and Holley 2002).

A variation in the process of cytokinesis, which normally produces two separate cells, in which cytokinesis is incomplete and the daughter cells remain connected by intercellular bridges (Hime et al. 1996; Ventelä et al. 2003; Greenbaum et al. 2006), occurs in gametogenesis in many organisms from Drosophila to vertebrates. Intercellular bridges persisting after incomplete cytokinesis also occur between the daughter cells of differential gonial mitoses in both sexes of Acricotopus (Fig. 1c, insert in Fig. 1d), thereby forming a special type of syncytium. Such bridges 
allow the transport of cytoplasmic components and even organelles from one cell to the other. In Drosophila females, the 15 nurse cells and one oocyte of an egg chamber are derived from a single germ-line precursor cell by four mitotic divisions. Cytokinesis during these mitotic cycles is incomplete, leaving cytoplasmic bridges, called ring canals, through which cytoplasm, including organelles, protein and RNA, are transported into the oocyte (Mahajan-Miklos and Cooley 1994). Such an intercellular transport of organelles via cytoplasmic bridges connecting early rat spermatids has also been reported for the male germ-cell-specific chromatoid body (Ventelä et al. 2003).

In Acricotopus, the aberrant spermatocyte receives the vast majority of the mitochondria as a result of the polar arrangement and unequal segregation. Fluorescence staining of live mitochondria in this study has given clear evidence for the transport of mitochondria from the aberrant spermatocyte through the cytoplasmic bridge to the primary spermatocyte. Cytoplasmic dynein is known to be involved in the active intracellular transport of mitochondria and other organelles along MTs and actin filaments (Mazurkiewicz and Kubrakiewicz 2001; Hollenbeck and Saxton 2005) and in the kinesin-1-driven slow and fast cytoplasmic streaming in Drosophila oocytes (Serbus et al. 2005). The translocation of mitochondria through the cytoplasmic bridge in Acricotopus may also be caused by an active dynein-mediated transport along MTs or by a kinesin-driven cytoplasmic stream or by a combination of both.

The cell-to-cell transport of cytoplasmic components, including mitochondria, might indicate a function of the aberrant spermatocyte similar to that of the nurse cell for the oocyte in the female, i.e. to nourish the primary spermatocyte or to supply it with specific components during growth until entry into meiotic divisions. Other functions supposed for the intercellular bridges in the germ line are the exchange of signals regulating synchronous meiotic cell divisions and the sharing of gene products in haploid germ cells (Guo and Zheng 2004; Greenbaum et al. 2006).

In early syncytial embryonic development in Acricoto$p u s$, all the Ks are eliminated from the future somatic nuclei during specific elimination mitoses (Staiber 2000, 2006). Thereafter, the somatic cells contain only Ss $(2 n=6)$. Only the pole cells, the germ line representatives, retain the Ks and carry them to the next generation. The monopolar movement of $\mathrm{Ks}$ in differential gonial mitoses produces one daughter cell receiving no Ks but only two S sets, resulting in the same chromosome constitution as the somatic cells. In other words, the aberrant spermatocyte and the nurse cell are germ-line descendants that become secondarily somatic with respect to their chromosome constitution.

The spindle plays a crucial role in differential mitosis. A first analysis of the sequential changes of the spindle MTs in differential mitosis of Acricotopus was performed on fixed spermatogonial cells by indirect immunofluorescence with an anti-beta-tubulin antibody (Staiber 1994). In the present study, the use of fluorochrome-labelled paclitaxel (taxol) on living cells has allowed a more detailed study of MT arrangements and of sequential changes of the spindle in differential mitosis. Paclitaxel is an MT-stabilising anticancer drug; it binds polymerised tubulin and promotes tubulin assembly (Diaz et al. 2003). Gamma-tubulin is present in the centrioles and, thus, the centrosomes are also labelled by paclitaxel. Normal gonial mitoses in Acricotopus exhibit equally sized half spindles, whereas differential mitoses show asymmetric spindle positioning and unequally sized half spindles. A similar asymmetric MT distribution, as detected in differential mitosis, also occurs during the asymmetric division of neuroblasts in Drosophila (Giansanti et al. 2001).

Thus far, uncertainties remain regarding (1) whether the unseparated $\mathrm{K}$ sister chromatids become mono-oriented by MT attachments at one or both sister kinetochores, i.e. with monotelic or syntelic attachments (Maiato et al. 2004), (2) the way in which mono-oriented Ks arrange themselves in the metaphase plane and (3) the way that their synchronous monopolar migration is regulated. The general assumptions are that only an opposite polewards-directed MT occupancy of sister kinetochores will allow chromosomes to align in the metaphase plate and that the resulting tension exerted at the kinetochores upon bipolar attachment activates the anaphase-promoting complex/cyclosome (APC/C) and thus the onset of anaphase. In this way, sister chromatid separation is initiated by a separase-dependent pathway effecting the cleavage of centromeric cohesin (Yanagida 2005; Blow and Tannaka 2006). Recently, however, monooriented chromosomes have been shown to congregate at the metaphase plane by movement along kinetochore MTs of other previously equatorially arranged and bi-oriented chromosomes (Kapoor et al. 2006). In differential mitosis, the Ks could possibly use the MT fibres attached to the bioriented $\mathrm{S}$ sister kinetochores to congress at the metaphase zone. A spindle-assembly checkpoint delays the activation of the $\mathrm{APC} / \mathrm{C}$ in the presence of mono-oriented chromosomes in a normal mitosis (Musacchio and Hardwick 2002). If MTs are attached only at one K sister kinetochore, it is then essential for the successful monopolar movement of all Ks that the cleavage of centromeric cohesin takes place first, after the arrival of the Ks at the pole. Sister chromatid separation taking place too early during the polewards motion of the Ks could lead to a loss or a missegregation of Ks. Therefore, a feedback mechanism that is triggered when the Ks reach a specific position near the spindle pole has to be assumed for initiating $\mathrm{S}$ sister chromatid separation. Sequential anaphase movements of different sets of chromosomes within a cell, first as unseparated sister chromatids (the Ks) and then as single 
chromatids (the Ss), are unusual. These subsequent chromosome movements in the last gonial mitosis of Acricotopus may be regulated by the differential activation of kinetochore motor proteins or by the differential regulation of depolymerisation of kinetochore MTs (Maiato et al. 2004; Cameron et al. 2006).

The present study demonstrates that a mother cell in the germ line of Acricotopus performs a specific mitosis that produces two daughter cells with markedly different chromosomal constitutions and different developmental fates. The mother cell arranges its mitochondria and spindle MTs in asymmetrical but opposite positions. The mitochondria partition unequally to the daughter cells but are then transported back through a permanent cytoplasmic bridge from the aberrant spermatocyte to the primary spermatocyte. Of interest, in the future, will be the determination of whether the asymmetric distribution of mitochondria occurs concomitantly with a polar depositioning of cell-fate determinant proteins such as Miranda or Numb, which are known to be involved in the asymmetric division of neuroblasts in Drosophila, and whether other cytoplasmic components in addition to mitochondria are transported through the cytoplasmic canal connecting the daughter cells.

Acknowledgements The author is grateful to Prof. Anette Preiss for support, to Dr. Heiko van der Kuip (Dr. Margarete Fischer-Bosch Institute of Clinical Pharmacology, Stuttgart, Germany) for providing Oregon Green 488 paclitaxel and to the referees and Prof. Neil Jones for helpful suggestions and corrections to the manuscript.

\section{References}

Bauer H (1970) Rearrangements between germ-line limited and somatic chromosomes in Smittia parthenogenetica (Chironomidae, Diptera). Chromosoma 32:1-10

Bauer H, Beermann W (1952) Der Chromosomencyclus der Orthocladiinen (Nematocera, Diptera). Z Naturforsch 7b:557-563

Bellaiche Y, Gho M, Kaltschmidt JA, Brand AH, Schweisguth F (2001) Frizzled regulates localization of cell-fate determinants and mitotic spindle rotation during asymmetric cell division. Nat Cell Biol 3:50-57

Betschinger J, Knoblich JA (2004) Dare to be different: asymmetric cell division in Drosophila, C. elegans and vertebrates. Curr Biol 14:R674-R685

Blow JJ, Tanaka TU (2006) The chromosome cycle: coordinating replication and segregation. EMBO Rep 6:1028-1034

Cameron LA, Yang G, Cimini D, Canman JC, Kisurina-Evgenieva O, Khodjakob A, Danuser G, Salmon ED (2006) Kinesin 5independent poleward flux of kinetochore microtubules in PtK1 cells. J Cell Biol 173:173-179

Diaz JF, Barasoain I, Andreu M (2003) Fast kinetics of taxol binding to microtubules. J Biol Chem 278:8407-8419

Giansanti MG, Gatti M, Bonaccorsi S (2001) The role of centrosomes and astral microtubules during asymmetric division of Drosophila neuroblasts. Development 128:1137-1145
Greenbaum P, Yan W, Wu MH, Lin YN, Agno JE, Sharma M, Braun RE, Rajkovic A, Matzuk MM (2006) TEX14 is essential for intercellular bridges and fertility in male mice. Proc Natl Acad Sci USA 103:4982-4987

Guo GQ, Zheng GC (2004) Hypotheses for the functions of intercellular bridges in male germ cell development and its cellular mechanisms. J Theor Biol 229:139-146

Hime GR, Brill JA, Fuller MT (1996) Assembly of ring canals in the male germ line from structural components of the contractile ring. J Cell Sci 109:2779-2788

Hollenbeck PJ, Saxton WM (2005) The axonal transport of mitochondria. J Cell Sci 118:5411-5419

Horvitz HR, Herskowitz I (1992) Mechanisms of asymmetric cell division: two Bs or not two Bs, that is the question. Cell 68:237-255

Kaltschmidt JA, Brand AH (2002) Asymmetric cell division: microtubule dynamics and spindle asymmetry. J Cell Sci 115:2257-2264

Kapoor TM, Lampson MA, Hergert P, Cameron L, Cimini D, Salmon ED, McEwen BF, Khodjakov A (2006) Chromosomes can congress to the metaphase plate before biorientation. Science 311:388-391

Lopez de Heredia M, Jansen RP (2004) mRNA localization and the cytoskeleton. Curr Opin Cell Biol 16:80-85

Mahajan-Miklos S, Cooley L (1994) Intercellular cytoplasm transport during Drosophila oogenesis. Dev Biol 165:336-351

Maiato H, DeLuca J, Salomon ED et al (2004) The dynamic kinetochore-microtubule interface. J Cell Sci 117:5461-5477

Mazurkiewicz M, Kubrakiewicz J (2001) Intercellular cytoplasm transport during oogenesis of the moth midge, Tinearia alternata (Diptera: Psychodidae). Folia Biol 49:205-213

Musacchio A, Hardwick KG (2002) The spindle checkpoint: structural insights into dynamic signalling. Nat Rev Mol Cell Biol 3:731-741

Redi AA, Garagna S, Zacharias H, Zuccotti M, Capanna E (2001) The other chromatin. Chromosoma 110:136-147

Rivolta MN, Holley MC (2002) Asymmetric segregation of mitochondria and mortalin correlates with the multi-lineage potential of inner ear sensory cell progenitors in vitro. Dev Brain Res 133:49-56

Serbus LR, Cha BJ, Theurkauf WE, Saxton WM (2005) Dynein and the actin cytoskeleton control kinesin-driven cytoplasmic streaming in Drosophila oocytes. Development 132:3743-3752

Staiber W (1988) G-banding of germ line limited chromosomes in Acricotopus lucidus (Diptera, Chironomidae). Chromosoma 97:231-234

Staiber W (1994) Immunofluorescence study of spindle microtubule arrangements during differential gonial mitosis of Acricotopus lucidus (Diptera, Chironomidae). Cell Struct Funct 19:97-101

Staiber W (2000) Immunocytological and FISH analysis of pole cell formation and soma elimination of germ line-limited chromosomes in the chironomid Acricotopus lucidus. Cell Tissue Res 302:189-197

Staiber W (2006) Chromosome elimination in germ line-soma differentiation of Acricotopus lucidus (Diptera, Chironomidae). Genome 49:269-274

Staiber W, Behnke E (1985) Developmental puffing activity in the salivary gland and Malpighian tubule chromosomes of Acricotopus lucidus (Diptera, Chironomidae). Chromosoma 93:1-16

Thudium D (1974) Das Verhalten von Soma- und Keimbahnchromosomen bei Acricotopus lucidus Staeger (Orthocladiinae, Diptera). Doctoral thesis. University of Hohenheim

Ventelä S, Toppari J, Parvinen M (2003) Intercellular organelle traffic through cytoplasmic bridges in early spermatids of the rat: mechanisms of haploid gene product sharing. Mol Biol Cell 14:2768-2780

White MJD (1973) Animal cytology and evolution, 3rd edn. Cambridge University Press, Cambridge, pp 500-546

Yanagida M (2005) Basic mechanism of eukaryotic chromosome segregation. Philos Trans R Soc Lond B Biol Sci 360:609-621 